\title{
Parametric Design Process of Facade Elements with Characteristics of Fractal Geometry: Development, Evaluation and Application
}

XXIV International Conference of the Iberoamerican Society

\author{
Pedro Oscar Pizzetti Mariano \\ Universidade Federal de Santa Catarina | Brasil | pedro.pm@hotmail.com \\ Alice Theresinha Cybis Pereira \\ Universidade Federal de Santa Catarina | Brasil | alice.cybis.pereira@ufsc.br
}

\begin{abstract}
This article deals with the use of the characteristics of fractal linear geometry and the different knowledge related to the application of its patterns in architectural elements, considering their compositional potentialities. For this, a theoretical and practical trajectory was developed, such as understanding the characteristics of fractal geometry and the existing software and methods for its reproduction. As a result, a parametric process was developed that allows to recreate fractal patterns in architectural elements, making it possible to identify the necessary steps for their elaboration, identifying the potentialities of their use and the skills necessary to reproduce similar parametric processes.
\end{abstract}

Keywords: Fractal geometry; Dimension D; Parametric process; Design Process.

\section{INTRODUCTION}

This article deals with the use of the characteristics of linear fractal geometry applied in patterns of architectural elements. And it seeks to analyze the parametric process that makes this action possible, identify the stages of its elaboration, describe the potential of its use, and the skills necessary to reproduce similar processes. For this, a theoretical and practical trajectory was developed. Who's theoretical was analyzed the fractal patterns, their characteristics and applications, in addition to identifying software and processes that were used to reproduce the fractal characteristics. In the practical part, parametric programming was developed and the result was analyzed.

Using the patterns of nature during the design process can be an opportunity to approximate the construction, structure and geometry of the ornamentation and the user's scale. An example of this kind of connection between geometry, technology, and nature is the use of fractal components and rules through computer software that allow these patterns to be transformed into architectural ornaments. One of the possibilities for the insertion of fractal patterns is the use of computational design and tools, which allowed changes in the way architecture is conceived (SALINGAROS; MEHAFFY, 2007; SALINGAROS, 2003).

The possibility of generating patterns, establishing parameters and rules, directly influenced the composition and studies involving fractal geometry. Mandelbrot (2004) argues that theories of fractal geometry largely inspired the conception of twentieth-century architecture and generative design.

Mandelbrot (1977) states that a fractal is a structure that has invariance, regardless of the scale at which it is observed and is always linked to the same pattern or shape, keeping its original structure identical. The development of a fractal and its mathematical patterns are derived from four main characteristics: self-similarity; infinite complexity; irregularity or roughness; and a nonintegrin dimension.

This last fractal characteristic, a nonintegral dimension or dimension D, described by Mandelbrot (1977), can identify and measure various fractals. Many fractals do not contain easily identifiable patterns, and the method described by Mandelbrot, box counting, is a viable procedure. Spehar et al. (2003) describe that the fractal dimension or D can be determined by comparing the number of squares occupied in a mesh with different scales, where "D" is a value between 1.0 and 2.0 .

This feature is also associated with providing a positive psychological reading of the space around us. Taylor (2006) and Joye (2007) comment on a set of possible reasons that associate fractals with positive human interpretations. And unconsciously this may have influenced that the fractal geometry is present in architecture in their general context or in different details and volumes.

The use of recent digital tools and methods influences many professionals in various design situations, allowing, among other things, the development of sun protection elements with different and complex shapes. In some cases, these concepts are inspired by nature to develop their shapes and structures. The use of fractal geometry or its features, combined with the use of parametric algorithms, can assist in the creation and development of these complex concepts and shapes. Parametric processes also have the potential to encompass innovative concepts through the use of modeling and simulation. (MENGES, 2012; RIAM; ASAYAMA, 2016).

To apply the rules, characteristics and iterations of fractals, different software and methods can be used. In the research, we opted for the use of visual programming software and plug-ins. To model generative processes 
within the field of architecture and design, one of the software that is accepted for the development of the algorithms is the Grasshopper plug-in, a graphic editor connected to the Rhinoceros 3D modeler. It provides a variety of mathematical and geometric operations and commands and can change the built digital model. (KOLAREVIC, 2005).

Considering these concepts, this paper, which is part of a master's dissertation, investigates the development of a process to develop, organize and evaluate a composition of facade elements with characteristics of linear fractal geometry.

\section{VISUAL PROGRAMMING DEVELOPMENT}

The present study shows the construction of a parametric process that involves different methods and concepts, resulting in a compilation of unified procedures in the development of a process to develop, organize and evaluate a composition of facade elements with characteristics of linear fractal geometry. To achieve this goal, a series of theoretical and practical actions were proposed to broaden the knowledge of the subject, acquire skills to manipulate the tools and interpret their results. The theoretical stage identified the different subjects for understanding the process and manipulating the necessary software. And the practical step was developed through the development of a parametric and generative design process, carried out through a visual programming platform.

For the development of the digital process, the Rhinoceros3D 5 modeling software was used. Within this software, the MII (incremental interactive model) was used as a method to build the process described by Silva and Videira (2001). This method allowed each of the steps developed in the visual programming to act independently, without requiring the complete completion of the previous step, for starting next. The elaboration of the model and of each of its parts was also performed in the Grasshopper visual programming software and assisted by the Hoopsnake and Diva plug-ins.

The whole process was subdivided into different stages, each of which also presents its divisions. The first stage of the process is divided into two parts, the assembly of the environment and the construction of the building. The two parts contain a series of steps that result in a threedimensional environment with nine parameterized blocks, with constructions, urban infrastructure and a space where a testing building will be inserted and in which the facade elements will be applied.

At the first step, a three-dimensional urban environment was developed to simulate an environment suitable for parametrical modifications. With this is crated a set of nine orthogonal urban blocks is formed, where it is possible to manipulate the topography, the number of lots per block (individually), the infrastructure (pathways), and the different characteristics of the constructions and scales. In the second stage of the development of the environment, a construction is modeled and inserted which will receive the compositions of the panels, at the previously designated site. In the end of this steps, a building model is created that is parametrically adjustable and susceptible to the insolation consequent to its surrounding urban environment, that also is parametrically modifiable.

For the research was used lot number seven in the central block (number five) to insert the test building, the site has the dimensions of $37 \mathrm{~m} \times 23.05 \mathrm{~m}$ and was directed to the North. The three-dimensional model to the test was molded from a central point on the chosen area, being possible to change different parameters as: width of the tested; length; floor height; width of slabs; and number of floors.

The composition of the urban environment has some limitations, such as: it is only possible to manipulate and modify nine blocks $(3 \times 3)$; one can modify its general dimensions, but orthogonally, without changing the perpendicular angles or using curved drawings; the dimensions of the blocks are generated from the division of the terrain and the sizing of the infrastructure. In the figure 1 and 2 presents the two environments used for research with the insertion of the construction in the middle of the center block.

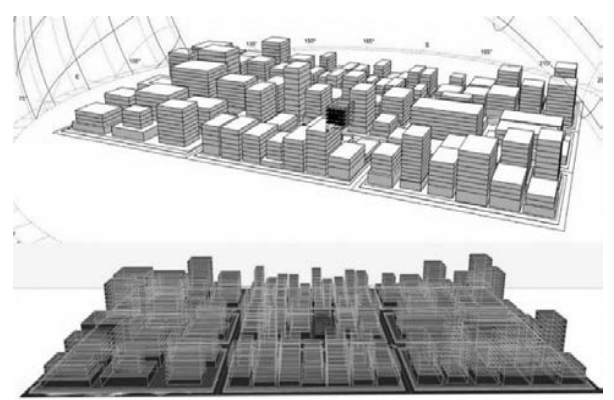

Figure 1: Parametrically adjusted urban environment to have level relief and constructions below $35 \mathrm{~m}$, seeking to maintain the central block of the urban environment provided with daylight.

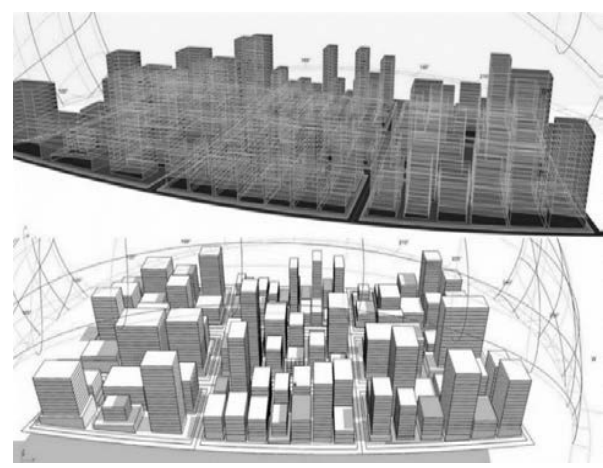

Figure 2: Parametrically adjusted urban environment to have uneven relief and constructions above $30 \mathrm{~m}$, seeking to increase the area of the central area of the model.

In the sequence the facade elements with fractal characteristics, that composed the main façade of the modeled building were developed. This stage of the process is divided into three parts: the design of panels with elements of fractal geometry; the analysis and selection of 
these panels and their respective iterations; and the optimization of the main elements.

The first stage of the confection and modeling of the panels with fractals is divided into two parts: the development and iteration rule for the façade elements; and the twodimensional modeling of these elements. For the development of each of the panels were used ten rules of Linear fractals, such as Square of Gasket; Jerusalem Cross; Sierpinski carpet; Cantor Set; Sierpinski Triangle; Minkowski's curve; Peano's curve; Dragon Curve; Fracta Tree; and Koch Island. Two examples of those rules can be observed by figure 3 and 4 , which presents the formation pattern for the fractal Cantor Set and Minkowski Curve (MARIANO; PEREIRA; VAZ, 2018).

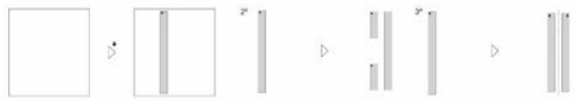

Figure 3: Example of application of fractal rules for the Cantor Set.

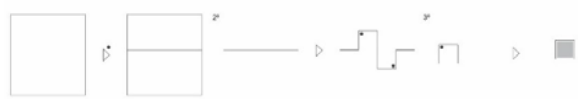

Figure 4: Example of application of fractal rules for the Minkowski Curve.

In the second step, the rules for generating the fractals were inserted into standard panels of $3 \mathrm{~m} \times 3 \mathrm{~m}$, and iterated four times, to generate five distinct panels, each with a different level of iteration. Thus, five different panels were generated for each type of fractal, totaling 50 panels. Each group of panels has its first facade element completely opaque, with no subtraction, and as iterations undergo, each rule subtracts a certain area. The Hoopsnake plug-in was used for modeling those patterns, which allowed iteration of the rule of each fractal pattern over and over again. As an example of the construction of these patterns the figures 5 and 6 presents the result of the modeling of the facade elements to the Cantor Set and Minkowski until its fourth iteration (MARIANO; PEREIRA; VAZ, 2018).

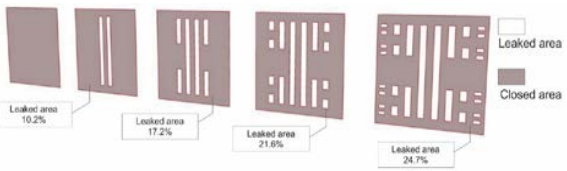

Figure 5: Panels with the Cantor Set, with percentage of the cast area for each iteration.

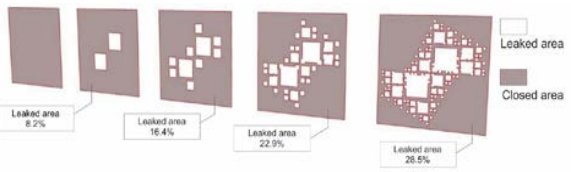

Figure 6: Panels with the Minkowski Curve, with percentage of the cast area for each iteration.

The panels modeled with fractal geometry designs were submitted to different daylights tests, whose objective was to identify the results of the luminous behavior of each panel group and to verify the different iterations of each pattern. This evaluation of the daylight behavior, allowed to identify and selected the patterns with the best daylight performance (MARIANO; PEREIRA; VAZ, 2018).

To analyze the light results of each panel, a virtual environment was modeled with the following dimensions: $6 \mathrm{~m}$ deep, $6 \mathrm{~m}$ wide and $3 \mathrm{~m}$ high. The model was adjusted so that the facade could be composed of a total of 8 panels, each of them as the same dimension $(1.5 \times 1.5 \mathrm{~m})$. The indexes, used to verify the light behavior of the environment, followed the models of the IES (Illumination Engineering society), and three indexes were measured: Useful Daylight Illuminance (UDI), SDA (spacial daylight autonomy) and ASE (annual sunlight exposure). Dynamic simulations were done using the DIVA as a plug-in for Grasshopper and using the BRA_FLORIANOPOLIS838990_SWERA climatic archive was used, referring to the city of Florianópolis, SC, Brazil. Each composition was evaluated twice in different positions. In total, 100 simulations were run, ten for each fractal geometry pattern (MARIANO; PEREIRA; VAZ, 2018).

And the panels that presented better daylight performance through the daylight selection capability, in different proportions for each iteration, were the elements developed with the fractal rules: Sierpinski Carpet, Minkowski Curve, Dragon Curve and Singer Set. These elements have demonstrated that they have different light filtrations for each iteration (MARIANO; PEREIRA; VAZ, 2018).

The panels that presented better luminous performance through the daylight selection capability, in different proportions for each iteration, were the elements developed with the fractal rules: Sierpinski Carpet, Minkowski Curve, Dragon Curve and Singer Set. These elements have demonstrated that they have different light filtrations for each iteration. (MARIANO; PEREIRA; VAZ, 2018).

Afterwards, the four panels identified in the previous step received lighting control elements, and had their sizing adjusted to evenly distribute daylight in the indoor environment. This stage is divided in two others: the development and modeling of the components; and its simulation in a controlled environment through a generative process, with the Galapagos component.

The first part of this process step is the modeling and angulation of the different thicknesses that will complement the panels. Each panel was complemented by a unique composition of the process, since the different characteristics of each one of the fractals need specific alterations so that its parameters are modified. The alterations comprise the opening angle of the shelves and their respective length. Those characteristics can be observed by the figure 7 . 


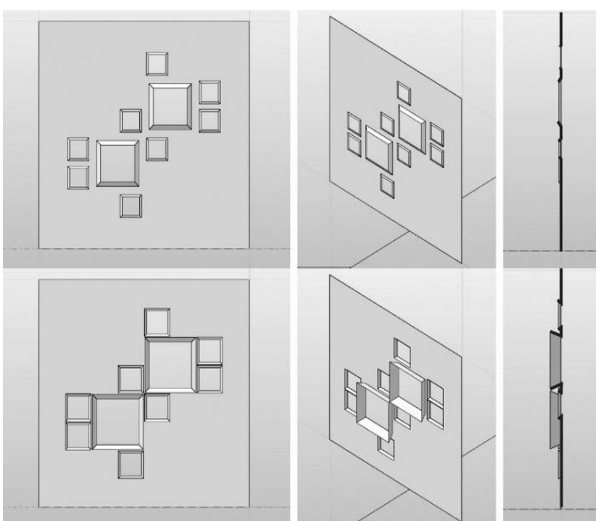

Figure 7: Different views of possible changes in the thickness of the facade element (open or closed opening angle and extended or short thickness)

When they are ready, the shelves will be optimized through the Galapagos component. The optimizations refer to the distribution of internal light in the environment (UDI) and the amount of radiation that passes through each of the solar protection elements.

In order to use the Galapagos component, it was necessary to model a simulation environment similar to the previous step, with the dimensions of six meters by six meters and three meters of height $(6 \mathrm{~m} \times 6 \mathrm{~m} \times 3 \mathrm{~m})$. The North facade was divided into a mesh of three by six, allowing the insertion of 18 elements equal of one meter by one meter. The simulations, more time, were performed through the DIVA and the climatic file used again was the BRA FLORIANOPOLIS838990 SWERA.

To equalize the values of the daylight indices, in order to optimize the results, the recurring responses obtained from the simulations were numeralized. In this process, the result of the simulations is transformed into a rational number between 0 and 1.0. As the Galápagos can only evaluate a quantitative response, the two resulting values had to be equivalent, assigning a percentage of $50 \%$ for each one. Thus, the Galápagos could obtain the maximum value of 1.0 and the simulations of IDUs would reach 0.5 and those of amount of radiation 0.5 . For each result does not exceed the desirable value, UDI $75 \%$ and amount of radiation $55 \%$, wrongly balancing the values, when the result exceeds the ideal values, it is replaced by 0 , decreasing its final value.

In the third part of the process, the union of the two previous stages takes place. This stage is divided into two phases, the organization and division of the facade and the insertion and control of these elements. And it starts with the division and organization of the spaces that will be filled by the façade elements developed. Firstly, the facade of the teste building is divided, individualizing the facade of each floor, then the facade of the floors is divided into four equal parts. Next, each of these four spaces is divided again by an array, allowing the adjustment of the quantity and size of the facade elements. For this research it was decided to subdivide each part into sixteen smaller slices $(4 \times 4)$, totaling 64 facade elements per floor, with $1.13 \mathrm{~m}$ wide and $1,125 \mathrm{~m}$ high (figure 8 ).
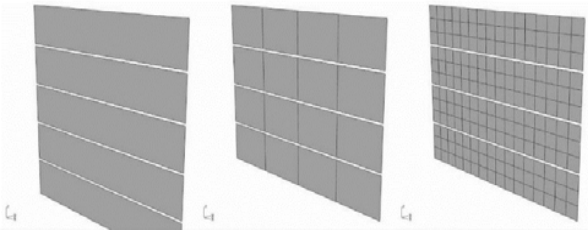

Figure 8: Result of the division of facades

Each of these spaces is tuned to receive one of the five iterations of a particular fractal pattern, developed in the previous steps. The fractals are updated to occupy one of the positions of the matrix of each floor, automatically adjusting its size (width, length and depth) to the division made before, and positioning itself, initially, from the reading of the radiation in the facade.

For the analysis of radiation, the DIVA plug-in was again used, evaluating, in this case, the average radiation incident on each floor. The panels are organized by dividing their different iterations according to the intensity of the radiation incident on the facade, placing the panels with more iterations (more subtraction of the shape) in the quadrants where the radiation indices are smaller, and the panels with less iterations (less subtraction in shape) in the divisions with higher incidence of radiation. Figure 9 shows an example of this sequence of actions. It is possible to control the positioning of the different iterations by means of a value window. These windows, in turn, make it possible to adjust the intervals that each type of panel occupy.

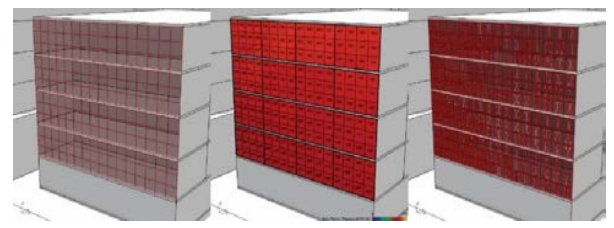

Figure 9: Result of the fill sequence.

After the organization of the panels, the main curves that compose them are mapped and extracted, this selection is made to contemplate the count of the fractal dimension of the composition. This mapping of the curves that make up the facade elements is automatic and updated to each type of fractal used or the composition developed.

Finally, the fractal dimension of the composition of the panels is verified through box-counting. For this part of the process, the curves that make up the panels are superimposed on a mesh that fills the space used for the facade elements. The process is performed three times and averaged in a four by four $(4 \times 4)$, eight by eight $(8 \times 8)$ matrix and a twelve by twelve $(12 \times 12)$ matrix. With this Counting it is possible to identify if the composition has a fractal dimension. If the result of the box-counting is upper of 1.0 the compositions are fractal, and if the values are closer or between of 1.3 and 1.5 the composition possible bring positive responses to human perception. 


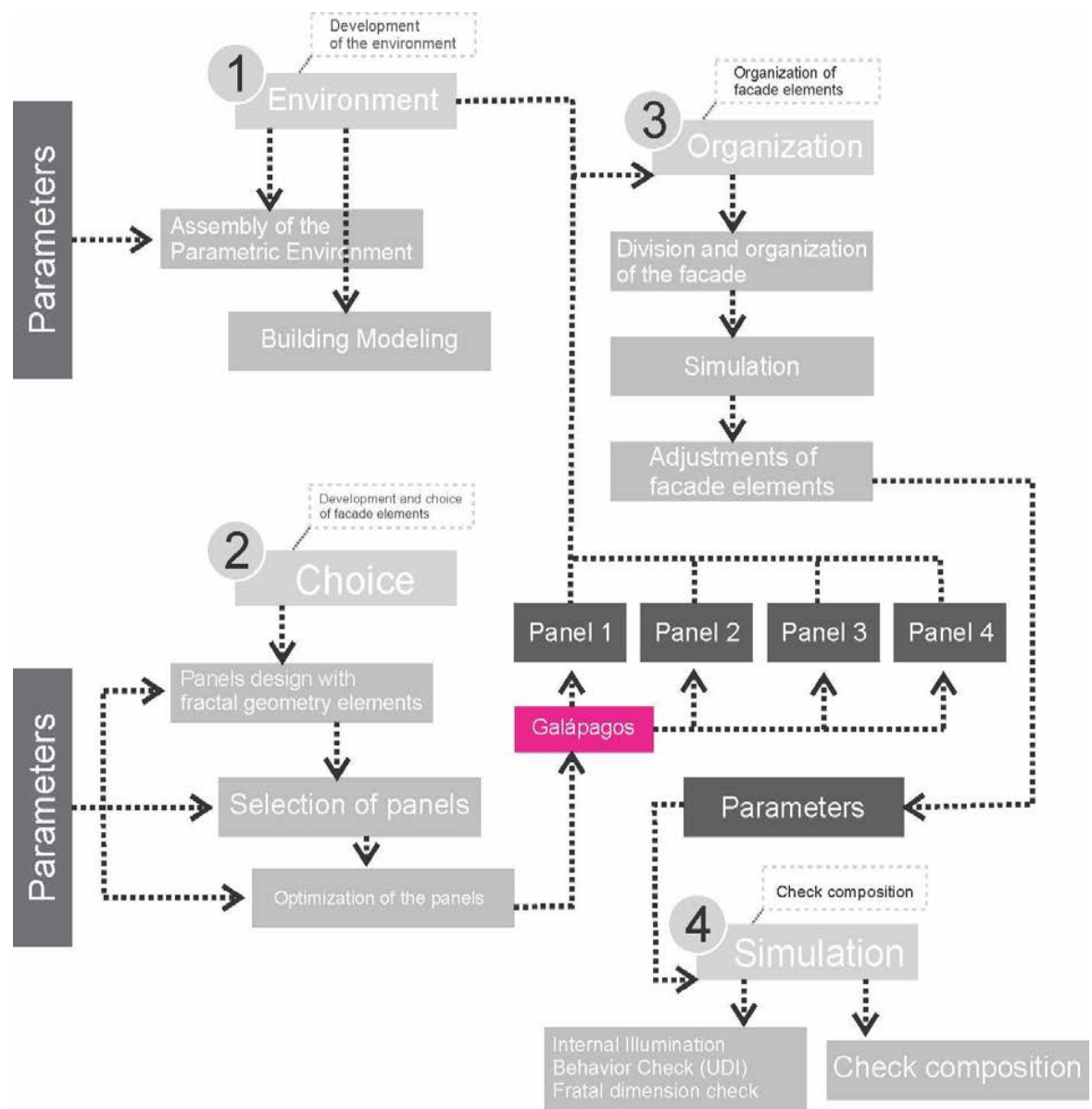

Figure 11: Structure of the process and its logical sequence.

\section{RESULTS}

As a result of the parametric process, elements, arrangements and simulations were created, able to develop, potentiate and organize different geometric elements with geometric characteristics of linear fractals. The elements created by this process can receive different attributions when used in façades, becoming an instrument of identity and composition that looks for possible positive psychological responses as well as element of shading and distribution of internal natural light.

During the process verification step, the four different types of panels were organized with the same compositional arrangement. It is possible to verify which of the four fractal patterns present the fractal dimension indices closer to the range of $1.3 \mathrm{D}$ to $1.5 \mathrm{D}$. The results of these organizations can be observed in Table 1.

Table 1: Results of the fractal dimension.

$\begin{array}{ccccc}\begin{array}{c}\text { Fractal } \\ \text { type }\end{array} & \begin{array}{c}\text { Minkowski } \\ \text { curve }\end{array} & \text { Cantor Set } & \begin{array}{c}\text { Dragon } \\ \text { Curve }\end{array} & \begin{array}{c}\text { Sierpinski } \\ \text { Rug }\end{array} \\ \text { D. Fractal } & 1.08 \mathrm{D} & 1.12 \mathrm{D} & 1.14 \mathrm{D} & 1.25 \mathrm{D}\end{array}$

The numerical interval, used for this first organization, was adjusted to seek a fractal dimension greater than $1.3 \mathrm{D}$ and to allow the visual contemplation of the environment from the internal environment. However, in the results of this first verification (fractal dimension), it can be seen that even with a larger range for the values, which correspond to the panels with more openings (more iterated), the filling of the spaces by box-counting was not enough so as to reach a size greater than $1,3 \mathrm{D}$.

Evaluating these organizations, it was observed that different composition intervals are necessary for each fractal pattern, since their differentiated characteristics and patterns make their results unique, having a fractal dimension dependent on the rule and the design that is chosen. To compare the results in terms of composition, an image with the two arrangements developed was humanized, representing the end result of the process (figure 10) 


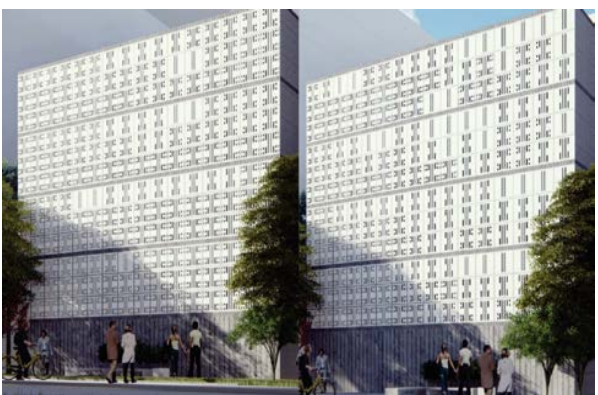

Figure 10: Humanization of two compositions made with the parametric process, arranged by different radiation indices that affect the façade.

Also were identify and characterized the steps to develop the parametric process capable of creating, organizing and evaluating elements of facade with fractal characteristics, four different stages were identified in chronological form, named as: environment; choice; organization; and simulation. Figure 11 represents the general structure of the parametric process

\section{CONCLUSION}

With the parametric process was possible to identify some conclusions. The first is the identification of the steps of the process resulted in the identification of four major stages that compose it: Environment, Choice, Organization and Simulation. Each stage was developed to complete a singular goal, allowing different changes not to influence the continuity of the whole. The first step performed (Environment) enables you to create parametric environment and building. The second stage (Choice) develops panels by means of fractal rules, allows to choose the panels with greater potential, and to add elements and to evaluate them by means of a genetic algorithm. The next step (Organization) joins the previous two steps, in addition to the façade elements. And the last phase (Simulation), verifies this organization resulting from the complete process.

This sequence of stages presents different assumptions for other uses. They have also been shown to be a coherent sequence for the development of methods and processes involving fractal geometry, using other features and patterns of fractal geometry with other purposes like structure, comfort, etc. Or in the construction of different parametric processes, involving various forms, uses and indices (thermal, acoustic, sensorial, physical, etc.).

The potential of the use of fractal geometry as a basis for the design of different sunscreens allows controlling the behavior of natural light within the internal environment through its different iterations. The different adjustments made in the step of choosing also allowed to extend the potential of this geometry in the construction of solar protectors, allowing to develop variations for fractal design, to create more iterations and to adjust the shelves of light.

Another conclusion reached in the research is about the development and handling of the process, considering that it requires a series of specific knowledge (such as: programming, logical processes, simulation and others), which if not used, may compromise the interpretation of the indexes or do not approve its use.

To develop geometries, which present features of fractal geometry, with different architectural purposes, it is necessary to deepen the knowledge about the discipline that will guide its creation and evaluation. So that the other steps of the process (Organization and Simulation) have results without errors.

The work presented limitations, in different stages, related to the processing capacity of the hardware used to make the simulations. During the use of Galapagos, the time of the simulations limited, in some types of fractals, the number of families generated by the plug-in. In counting the box counting, the time to count the filled spaces also prevented the development of a greater number of compositions. The delay in obtaining the different results of the process, such as the internal simulations and the fractal dimension, resulted in difficulties in data collection. At different times, the process needed to be repeated a few times for the reading of the indices and figures to be accurate.

\section{REFERENCIAS}

JOYE, Yannick. Fractal Architecture Could Be Good for You. Nexus Network Journal, [s.I.], v. 9, n. 2, p.311-320, out. 2007 Springer Nature. http:/dx.doi.org/10.1007/s00004-007-0045-y.

KOLAREVIC, B. Architecture in the Digital Age: Design and Manufacturing. [s.1.]: Taylor \& Francis Group, 2005. 320 p.

MANDELBROT, Benoit B. The Fractal Geometry of Nature. Nova lorque: W. H. Freeman and Company, 1977. 480 p.

MANDELBROT, Benoit. Fractals and Chaos: The Mandelbrot Set and Beyond. [s.1.]: Springer, 2004. 308 p.

MARIANO, Pedro Oscar Pizzetti; PEREIRA, Alice Theresinha Cybis; VAZ, Carlos Verzola. Avaliação luminosa de elementos de fachada com características fractais. Parc Pesquisa em Arquitetura e Construção, [s. I.], v. 9, n. 1, p.3-18, 31 mar. 2018. Universidade Estadual de Campinas http://dx.doi.org/10.20396/parc.v9i1.8650255.

MENGES, Achim. Biomimetic design processes in architecture: morphogenetic and evolutionary computational design. Bioinspiration \& Biomimetics, [s. I.], v. 7, n. 1, p.1-11, 16 fev. 2012. IOP Publishing. http://dx.doi.org/10.1088/1748$3182 / 7 / 1 / 015003$.

RIAM, lasef Md; ASAYAMA, Shuichi. Computational Design of a nature-inspired architectural structure using the concepts of self-similar and random fractals. Automation in Construction, [s.l.], v. 66, p.43-58, jun. 2016. Elsevier BV. http://dx.doi.org/10.1016/j.autcon.2016.03.010.

SALINGAROS, N. A. (2003). The sensory value of ornament. Communication \& Cognition, 36 (3-4), 331-351.

SALINGAROS, Nikos A.; MEHAFFY, Michel W. A Theory of Architecture. [s.I.]: Intercollegiate Studies Inst, 2007. 278 p.

SILVA, Alberto Manuel Rodrigues da; VIDEIRA, Carlos Alberto Escaleira. UML - Metodologias e Ferramentas CASE: Linguagem de Modelação UML, Metodologias e Ferramentas CASEM na Concepção e Desenvolvimento de Software. Portugal: Edições Centro Atlântico, 2001. 578 p.

SPEHAR, Branka et al. Universal aesthetic of fractals. Computers \& Graphics, [s.I.], v. 27, n. 5, p.813-820, out. 2003. Elsevier BV. http://dx.doi.org/10.1016/s0097-8493(03)00154-7.

TAYLOR, R.p. Reduction of Physiological Stress Using Fractal Art and Architecture. Leonardo, [s. I.], v. 39, n. 3, p.245-251, jun. 2006. MIT Press - Journals. http://dx.doi.org/10.1162/leon.2006.39.3.245. 